\title{
Follow-Up Study of the Cardiopulmonary and Psychological Outcomes of COVID-1 9 Survivors Six Months After Discharge in Sichuan, China
}

\author{
Shuiping Dai $\mathbb{D}^{1, *}$ \\ Bennan Zhao ${ }^{2, *}$ \\ Dafeng Liu ${ }^{2}$ \\ Yongzhao Zhou ${ }^{3}$ \\ Yaling Liu ${ }^{2}$ \\ Lijuan Lan $^{2}$ \\ Yalun $\mathrm{Li}^{3}$ \\ Wenxin $\mathrm{Luo}^{3}$ \\ Yilan Zeng ${ }^{2}$ \\ Weimin $\mathrm{Li}^{3}$ \\ 'Center of Gerontology and Geriatrics, \\ West China Hospital, Sichuan University, \\ Chengdu, Sichuan, People's Republic of \\ China; ${ }^{2}$ Department of Comprehensive \\ Internal Medicine, The Public and Health \\ Clinical Center of Chengdu, Chengdu, \\ Sichuan, People's Republic of China; \\ ${ }^{3}$ Department of Respiratory and Critical \\ Care Medicine, West China Hospital, \\ Sichuan University, Chengdu, Sichuan, \\ People's Republic of China \\ *These authors contributed equally to \\ this work
}

Purpose: Some studies have shown that patients with coronavirus disease 2019 (COVID19) still have sequelae after discharge. However, little is known about the long-term physical and psychological sequelae of patients, especially factors that influenced the prognosis.

Patients and Methods: Patients with COVID-19 were followed up for 6 months. The psychological status of patients was evaluated by DASS-21 questionnaire, while physical functions were determined using medical history, laboratory examination, thoracic computed tomography (CT), and echocardiography.

Results: Fifty patients infected with COVID-19 were enrolled, and 11 (22\%) patients still showed symptoms related to COVID-19. The mean contents (cells/ul) of CD3+ cells, CD4+ and CD8+ T, B lymphocytes and NK cells of the survivors elevated significantly after 6 -month discharge $(\mathrm{P}<0.001)$. The frequency of ground-glass opacities and consolidations decreased from $90 \%$ to $42 \%(\mathrm{P}<0.001)$, and $54 \%$ to $20 \%$, $(\mathrm{P}=0.001)$, respectively, while the changes of reticulation and bronchiectasis were insignificant $(P>0.05)$. The frequency of left ventricular diastolic dysfunction decreased from $40 \%$ to $15 \%(\mathrm{P}=0.002)$. Depression was observed in $5(12.5 \%)$ participants, stress in $3(7.5 \%)$, anxiety in $6(15 \%)$, and among them $1(2.5 \%)$ showed extremely severe anxiety. Covariation analysis elucidated age might be a risk factor (OR: $1.09,95 \% \mathrm{CI}$ : $1.01-1.18, \mathrm{P}=0.038)$, while $\mathrm{NK}$ cell was a good prognostic factor for pulmonary recovery. The comorbidities were significantly positive correlated with persist pulmonary damage $(\mathrm{r}=0.33, \mathrm{P}=0.020)$. Compared with patients with antiviral therapy, patients without antiviral therapy had higher anxiety score ( 3 vs $0, P=$ 0.033).

Conclusion: After 6-month discharge, the persisting cardiopulmonary damage was observed in recovery patients, and psychological implications should not be ignored. Age, comorbidities, NK cell and antiviral therapy might be associated with the prognosis of COVID-19.

Keywords: COVID-19, follow-up, cardiopulmonary, psychology, influence factors

\section{Introduction}

Coronavirus disease 2019 (COVID-19) is a public health emergency of international concern and its pathogen named severe acute respiratory syndrome coronavirus 2 (SARS-CoV-2). ${ }^{1}$ The SARS-CoV-2 virus is genetically closely related to severe acute respiratory syndrome coronavirus (SARS-CoV) and they all induced imbalanced host response and caused severe pulmonary pathology, which made the similar clinical and mechanistic characteristics. ${ }^{2-6}$ Previous follow-up studies on SARS have shown that survivors still have residual lung lesions at 3,6 months and one year after disease
Correspondence: Weimin Li; Yilan Zeng Email weimin003@163.com; 2499081791@qq.com 
onset, such as pulmonary fibrosis and ground-glass opacities (GGO), ${ }^{7,8}$ subclinical diastolic impairment, ${ }^{9}$ and reported elevated levels of psychological stress. ${ }^{10}$ At present, the global epidemic situation of COVID-19 has not been effectively controlled. The follow-up of recovery patients is helpful for us to understand the sequelae of SARS-CoV-2 infection, so as to guide the treatment and long-term management of patients with COVID-19. ${ }^{11}$ A previous 2-week follow-up study showed that SARS-CoV-2 was significantly reactivated in discharged patients. ${ }^{12}$ For long-term investigations, it has been reported that some patients with COVID-19 still have abnormal physiological functions including cardiopulmonary function at 1 month and 3 months after discharge, ${ }^{13,14}$ liver injuries at 40 days after discharge, ${ }^{15}$ and striking decreased health-related quality of life (HRQoL) score compared with population norm. ${ }^{16}$ Meanwhile, obvious post-traumatic stress disorder (PTSD) of the survivors were identified by previous investigations. Ten percent patients reported depression at one-month postdischarge, ${ }^{17}$ and a previous 6-month follow-up study found COVID-19 survivors were mainly troubled with anxiety or depression and severely patients had more severe pulmonary diffusion capacities. ${ }^{18}$ However, the effect of hospitalization treatments on pulmonary rehabilitation and the recovery of cardiac function remains unclear, which limits the valid management for the recovering patients after discharge. We thus designed this prospective observational follow-up study to characterize 6-month outcomes of recovery patients with COVID-19, including symptoms, immunocytes, SARS-CoV-2 antibodies, cardiopulmonary damage, psychological status.

\section{Materials and Methods \\ Patients and Study Design}

This study has been approved by Ethics Committee of the Public Health Clinical Center of Chengdu (approval number: PJ-K2020-06-01) and registered at China Clinical Trial Registration Center (registration number: ChiCTR2000034563). Informed consent was obtained from each participation. This was a prospective observational follow-up study of recovery COVID-19 patients followed up between January 29 and October 13, 2020 in the Public Health Clinical Center of Chengdu, Sichuan, China. Inclusion criteria for patients with COVID-19: (1) local patients diagnosed in Sichuan Province from January 2020 to March 2020; (2) age $\geq 18$ years; (3) 6-month follow-up was completed. Exclusion criteria: (1) refused to join the study or sign informed consent. Fifty patients were followed up 6 months after discharge. All patients diagnosed with COVID-19 was confirmed if a typical clinical presentation along with a positive RTPCR SARS-CoV-2 test obtained from a nasopharyngeal or oropharyngeal swab were present. ${ }^{19}$ The criteria for discharge and disease severity status were defined by Diagnosis and Treatment Program of New Coronary Pneumonia 7th ed ${ }^{20}$ National Health Commission of the People's Republic of China.

\section{Materials}

The physical effects of patients were evaluated by asking medical history, laboratory and imaging examinations. The general information of the subjects and the following indicators were collected: (1) symptoms; (2) laboratory examination indexes, such as $\mathrm{C}$ reactive protein, $\mathrm{T}$ lymphocyte subsets, glycated haemoglobin A1c (HbAlc) and D-dimer; (3) echocardiography; (4) chest Computed Tomography. Serum IgM and IgG antibodies against SARS-CoV-2 were tested at 6 months after discharge.

The psychological status of patients 6 months after discharge was evaluated by Depression Anxiety Stress Scale (DASS-21), ${ }^{21}$ which was a 4-point Likert-type scale that measured the negative emotional (Depression, Anxiety and Stress) stated experienced during the last week through 21 items. Chinese version of the DASS-21 was used in this study. ${ }^{22}$ The scores for each of the three components were calculated by summing up the scores for the relevant items, and because the DASS- 21 is a short form version of the DASS (the Long Form has 42 items), the final score of each item groups multiplied by two.

\section{Statistical Analysis}

Kolmogorov-Smirnov test was used to analyze the distribution of continuous variables. Normal distribution variables at discharge and 6 months after discharge were compared by paired $t$-test and represented by mean (standard deviation), non-normal distribution variables by Wilcoxon signed-rank test and represented by median (inter-quartile range, IQR). For the comparison of two independent samples, $t$-test was used for continuous variables of normal distribution, and Mann-Whitney test was used for non-normal distribution variables. CT and echocardiography findings were compared by $\mathrm{McNemar}$ test. In the correlation analysis, Logistic regression test was used when the dependent variable was classified variable; Spearman rank correlation was used for two quantitative variables; Pearson contingency coefficient 
test was used to analyze the correlation between the two categorical variables. P value $<0.05$ was considered to define statistical significance. All analyses were performed by SPSS 22.0 software (Chicago, IL, USA).

\section{Results}

\section{The Clinical Characteristics of Patients with COVID-196 Months After Discharge}

Fifty patients with COVID-19 were included in this study, aged from 25 to 80 years (mean age, $48 \pm 14$ years), and $25(50 \%)$ male patients. All patients were negative for RTPCR SARS-CoV-2 test 6 months after discharge. Eleven $(22 \%)$ patients have been diagnosed with severe or critical severe diseases, and $11(22 \%)$ patients still presented symptoms after 6-month discharge. Eighteen (36\%) patients had comorbidities, and the most common was hypertension $9(18 \%)$ and diabetes $8(16 \%)$. The decrease of activity tolerance was the most common signs (9 $(18 \%))$, which was manifested in the feeling of heart tired and tight breath after walking slowly for 400-600 meters, and needed to stop and rest, and without this symptom before SARS-CoV-2 infection. Five (10\%) patients had cough without sputum and $3(6 \%)$ had palpitations. Thirty-five $(70 \%)$ patients still presented SARSCoV-2 IgG antibodies positive after 6-month discharge, as shown in Table 1 . However, only $3(6 \%)$ patients after 6-month discharge showed positive SARS-CoV-2 IgM antibodies.

Of the 50 patients hospitalized in the Public Health Clinical Center of Chengdu, 48 (96\%) patients were treated with interferon, 36 (72\%) patients with antiviral drugs (oseltamivir, lopinavir/ritonavir, and darunavir/cobicistat). Two (4\%) patients diagnosed with critical disease received methylprednisolone therapy.

\section{Changes of Hematological Indexes in Patients Discharged for 6 Months}

As shown in Table 2, the serum level of inflammatory index C-reactive protein and serum amyloid A protein decreased 6 months after discharge (CRP: 3.65 (6.80) vs 0.50 (1.44) mg/ L, P $<0.001$; SAA: 8.12 (22.35) vs 5.00 (1.79) mg/L, P < $0.001)$. On the day of follow-up after 6 months, the platelet count was significantly $\left(200.04(82.31) \times 10^{9}\right.$ per L) less than that at discharge $\left(225.43(82.31) \times 10^{9}\right.$ per L) $(\mathrm{P}=0.004)$. The values of CK (112.56 (52.74) U/L) and CK-MB (9.60 (2.46) U/L) after 6-month discharge increased markedly than
Table I Demographics and Clinical Characteristics of Patients 6 Months After Discharge $(n=50)$

\begin{tabular}{|l|c|}
\hline Characteristics & \\
\hline Age, mean (SD) & $48(14)$ \\
\hline Sex & \\
Men & $25(50)$ \\
Women & $25(50)$ \\
\hline Disease severity status & \\
Mild/general & $39(78)$ \\
Severe/critical & $11(22)$ \\
Comorbidities & $18(36)$ \\
Hypertension & $9(18)$ \\
Diabetes & $8(16)$ \\
Cardiovascular diseases & $1(2)$ \\
Chronic obstructive pulmonary disorder & $2(4)$ \\
Chronic liver diseases & $5(10)$ \\
\hline Signs and symptoms & $1 \mathrm{I}(22)$ \\
Decreased activity tolerance & $9(18)$ \\
Cough & $5(10)$ \\
Palpitation & $3(6)$ \\
Night sweat & $1(2)$ \\
Dyspnea & $1(2)$ \\
\hline Antibodies against SARS-CoV-2 & $35(70)$ \\
IgM positive & \\
IgG positive & $38(96)$ \\
\hline Therapy & $36(72)$ \\
Interferon & $2(4)$ \\
Antiviral drugs & \\
Methylprednisolone & \\
\hline
\end{tabular}

Abbreviation: SD, standard deviation; Number (percentage).

that at discharge (CK at $50.57(26.96) \mathrm{U} / \mathrm{L}$ with $\mathrm{P}<0.001$, and CK-MB at 8.57 (2.63) $\mathrm{U} / \mathrm{L}$ with $\mathrm{P}=0.012$ ). The obvious increase of level of albumin was observed after 6-month discharge (39.79 (4.59) to 47.39 (2.71) g/L, $\mathrm{P}<0.001)$. The values of ALT, AST, TBIL, and LDH of the participants were in the normal range $(<37 \mathrm{U} / \mathrm{L},<40 \mathrm{U} / \mathrm{L},<20.5 \mathrm{umol} / \mathrm{L},<245$ $\mathrm{U} / \mathrm{L}$, respectively) at the day of 6-month discharge.

The value of urea increased from $3.58(0.93)$ to 5.34 (1.26) $\mathrm{mmol} / \mathrm{L}(\mathrm{P}<0.001)$, while creatinine did not show significant statistic increase $(\mathrm{P}=0.642)$. Seven patients showed normal urea $(<6.9 \mathrm{mmol} / \mathrm{L})$ at discharge, but abnormal value at 6-month follow-up. All of these 7 patients had no history of kidney disease, only one patient diagnosed with severe disease, and only one patient had COVID-19 related symptoms 6 months after discharge. The percentage of HbA1c elevated from 5.04 (0.73) to 
Table 2 Laboratory Results of Patients at the Time of Discharge and 6 Months After Discharge

\begin{tabular}{|c|c|c|c|}
\hline Indicators & Discharge & 6 Months & $P$ value \\
\hline C-reactive protein ${ }^{\#}, \mathrm{mg} / \mathrm{L}$ & $3.65(6.80)$ & $0.50(1.44)$ & $<0.001$ \\
\hline Serum amyloid A protein ${ }^{\#}, \mathrm{mg} / \mathrm{L}$ & $8.12(22.35)$ & $5.00(1.79)$ & $<0.001$ \\
\hline Lymphocyte count ${ }^{\#}, \times 10^{9}$ per $\mathrm{L}$ & $1.23(0.64)$ & $1.80(1.30)$ & $<0.001$ \\
\hline Platelet count, $\times 10^{9}$ per $\mathrm{L}$ & $225.43(82.31)$ & $200.04(82.31)$ & 0.004 \\
\hline $\mathrm{ALT}^{\#}, \mathrm{U} / \mathrm{L}$ & $28.00(22.25)$ & $20.00(19.25)$ & 0.065 \\
\hline $\mathrm{AST}^{\#}, \mathrm{U} / \mathrm{L}$ & $23.50(11.00)$ & $19.00(9.25)$ & 0.776 \\
\hline Albumin, $g / L$ & $39.79(4.59)$ & $47.39(2.7 \mathrm{I})$ & $<0.001$ \\
\hline TBIL, umol/L & $8.56(4.38)$ & $8.68(4.19)$ & 0.873 \\
\hline $\mathrm{LDH}, \mathrm{U} / \mathrm{L}$ & $184.33(48.23)$ & $174.74(30.36)$ & 0.188 \\
\hline CK, U/L & $50.57(26.96)$ & I $12.56(52.74)$ & $<0.001$ \\
\hline CK-MB, U/L & $8.57(2.63)$ & $9.60(2.46)$ & 0.012 \\
\hline Creatinine, umol/L & $62.88(17.17)$ & $63.56(15.11)$ & 0.642 \\
\hline Urea, $\mathrm{mmol} / \mathrm{L}$ & $3.58(0.93)$ & $5.34(1.26)$ & $<0.001$ \\
\hline $\mathrm{HbAlc} \%$ & $5.04(0.73)$ & $5.40(0.48)$ & $<0.001$ \\
\hline D-Dimer, ug/mL & $0.84(0.44)$ & $0.63(0.27)$ & 0.011 \\
\hline CD3, cells/ul & $840.26(344.73)$ & $128 \mid .06(4 \mid 3.76)$ & $<0.001$ \\
\hline CD4, cells/ul & $462.37(218.84)$ & $673.56(217.52)$ & $<0.001$ \\
\hline CD8, cells/ul & $313.50(163.74)$ & $514.78(264.02)$ & $<0.001$ \\
\hline CD4/CD8 & $1.73(0.93)$ & $1.55(0.79)$ & 0.001 \\
\hline B cell, cells/ul & $98.93(66.72)$ & 197.34 (| | 0.45$)$ & $<0.001$ \\
\hline NK cell, cells/ul & 144.13 (70.58) & $237.26(148.60)$ & $<0.001$ \\
\hline
\end{tabular}

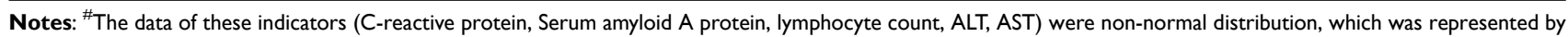
median (inter-quartile range), and Wilcoxon signed-rank test was used to compare the data. The data of other indicators (platelet count, albumin, TBIL, LDH, CK, CK-MB, creatine, urea, HbAlc, D-Dimer, CD3, CD4, CD8, CD4/CD8, B, NK) showed normal distribution, which was represented by mean (standard deviation), and the comparison of data used paired samples $t$ test. The $P$ value in bold indicated that the results were statistically significant.

Abbreviations: ALT, glutamic-pyruvic transaminase; AST, glutamic oxalacetic transaminase; TBIL, total bilirubin; LDH, Lactate dehydrogenase; CK, creatine kinase; CK-MB, creatine kinase isoenzyme; HbAlc, glycated haemoglobin Alc; NK, nature kill cells.

$5.40(0.48)(\mathrm{P}<0.001)$. Four patients showed normal HbA1c concentrations $(<6.07 \%)$ at discharge, but abnormal at 6-month follow-up. Among 4 of these patients, only one had diabetes, two diagnosed with severe disease, and one showed symptom at 6-month follow-up.

All immunocytes increased strikingly after discharge for 6 months (Table 2). The lymphocyte increased from 1.23 (0.64) to $1.80(1.30) \times 10^{9}$ per $\mathrm{L}(\mathrm{P}<0.001)$. The mean cell counts (cells/ul) of CD3+ cells, B lymphocytes and NK cells elevated after 6-month discharge than that at discharge (CD3: 1281.06 (413.76) vs 840.26 (344.73), P < 0.001; B: 197.34 (110.45) vs 98.93 (66.72), $\mathrm{P}<0.001$; NK: 237.26 (148.60) vs 144.13 (70.58), $\mathrm{P}<0.001)$, as shown in Figure S1A. $\mathrm{T}$ lymphocytes subsets, CD4+ and $\mathrm{CD} 8+$ cells, also increased 6 months after discharge $(\mathrm{P}<0.001$, Figure S1B) .

\section{Persistent Cardiopulmonary Damage and Its Influencing Factors}

Forty-eight patients completed chest $\mathrm{CT}$ examination in the hospital, of which 45 patients underwent the examination 6 months after discharge. Radiological lung abnormalities were still observed in 41 (91\%) patients. Some lung lesions decreased significantly 6 months after discharge, such as GGO from $90 \%$ to $42 \%$ and consolidation from $54 \%$ to $20 \%$ (Figure $1 \mathrm{~A}$ ). However, the frequency of reticular formation and bronchiectasis did not decrease significantly 6 months after discharge (Table S1).

To further elucidate the covariation relationship among the physical and psychological functions, the indicators of the follow-up patients, that might reflect the CT recovery of pulmonary, were analyzed by Pearson's continuity efficiency test and logistic regression analysis (Table 3). The presence of comorbidities was significantly positively correlated with persist pulmonary damage $(\mathrm{r}=0.33, \mathrm{P}=$ $0.020)$. Age was a risk factor for pulmonary recovery $(\mathrm{OR}=1.09,95 \% \mathrm{CI}: 1.01-1.18, \mathrm{P}=0.038) . \mathrm{NK}$ was a good prognostic factor for pulmonary recovery $(\mathrm{OR}=$ 0.98, 95\% CI: $0.97-0.99, \mathrm{P}=0.038$ ). Although no significant correlation between antiviral therapy and persist pulmonary damage (36 patients) was found $(\mathrm{r}=-0.15, \mathrm{P}=$ 0.296), the proportion of GGO and consolidation remarkably decreased from $94 \%$ to $36 \%(\mathrm{P}<0.001)$ and $56 \%$ to 
A

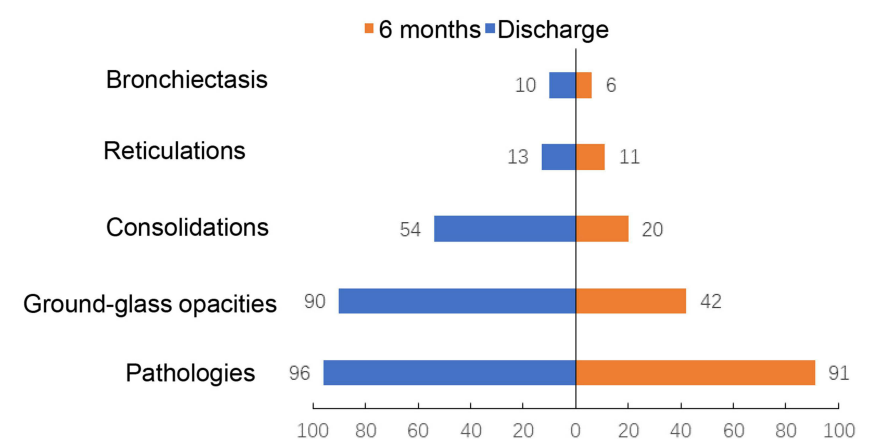

B

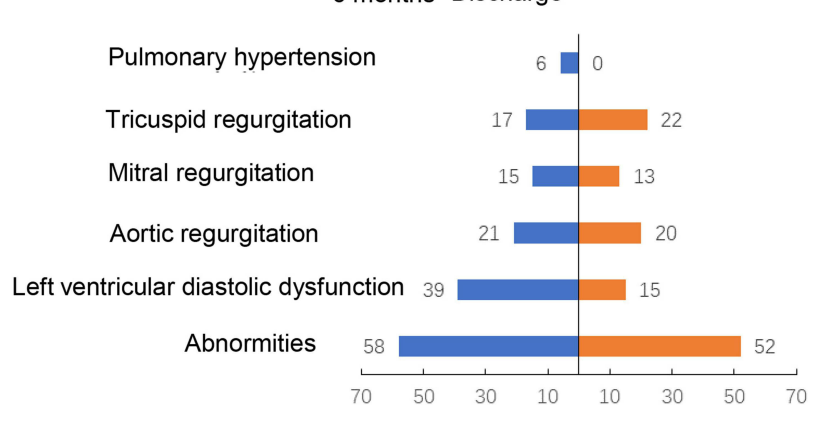

Figure I Percentage of lesions detected by chest computed tomography and echocardiography at discharge and six months after discharge. (A) Pathological findings assessed with computed tomography; (B) abnormities assessed with echocardiography.

$11 \%(\mathrm{P}<0.001)$, respectively. In 14 patients without antiviral drugs, the proportion of GGO decreased from $93 \%$ to $43 \%$ ( $\mathrm{P}<0.001)$, while no statistical difference was observed in consolidation $(\mathrm{P}=1)$.

Of the 50 patients, 48 completed echocardiography in the hospital and 46 at 6 months after discharge. The results showed obvious abnormalities found in 28 (58\%) patients in hospital, and in $24(52 \%)$ patients after 6-month discharge.
Nineteen $(40 \%)$ patients presented with a reduced left ventricular diastolic dysfunction in hospital. After 6-month discharge, only $7(15 \%)$ patients still presented with left ventricular diastolic dysfunction (Figure 1B). The frequency of aortic regurgitation, mitral regurgitation, and signs of pulmonary hypertension did not significantly change 6 months after discharge $(\mathrm{P}=0.878, \mathrm{P}=0.829, \mathrm{P}=0.532$, $\mathrm{P}=0.250$, respectively) (Table $\mathrm{S} 2$ ). No significant factors

Table 3 Analysis of Related Factors of Persist Cardiopulmonary Damage

\begin{tabular}{|c|c|c|c|c|}
\hline \multirow[t]{2}{*}{ Factors } & \multicolumn{2}{|c|}{ Pulmonary } & \multicolumn{2}{|c|}{ Heart } \\
\hline & $\mathbf{r}$ & $P$ & $\mathbf{r}$ & $P$ \\
\hline Male & 0.00 & 1.000 & 0.10 & 0.490 \\
\hline Comorbidities & 0.33 & 0.020 & -0.25 & 0.080 \\
\hline Severe/critical disease & 0.23 & 0.105 & -0.10 & 0.505 \\
\hline Antiviral therapy & -0.15 & 0.296 & 0.13 & 0.355 \\
\hline & OR $(95 \% \mathrm{Cl})$ & $P$ & OR $(95 \% \mathrm{Cl})$ & $P$ \\
\hline Age & $1.09(1.01-1.18)$ & 0.038 & $0.95(0.90-1.00)$ & 0.069 \\
\hline Urea & $0.86(0.38-1.92)$ & 0.712 & $0.53(0.25-1.13)$ & 0.099 \\
\hline $\mathrm{HbAIC}$ & $1.17(0.39-3.48)$ & 0.780 & $0.53(0.21-1.35)$ & 0.183 \\
\hline TBIL & $0.93(0.79-1.10)$ & 0.416 & $0.95(0.82-1.1 \mathrm{I})$ & 0.552 \\
\hline CK & $0.97(0.95-1.00)$ & 0.054 & $0.99(0.97-1.02)$ & 0.618 \\
\hline CD4 & $1.00(0.99-1.00)$ & 0.347 & $1.00(0.99-1.00)$ & 0.268 \\
\hline B & $1.00(0.99-1.01)$ & 0.731 & $0.99(0.98-1.00)$ & 0.145 \\
\hline NK & $0.98(0.97-0.99)$ & 0.016 & $1.00(0.99-1.01)$ & 0.969 \\
\hline
\end{tabular}

Notes: The correlation between the two classification variables was analyzed by Pearson continuity efficiency test. Univariate logistic regression analysis was used to study the influencing factors (continuous variables) of cardiopulmonary recovery. The $P$ value in bold indicates that the results were statistically significant.

Abbreviations: r, Pearson correlation; OR, odds ratio; Cl, confidence interval; CK, creatine kinase; HbAlc, glycated haemoglobin Al c; TBIL, total bilirubin; NK, nature kill cells. 

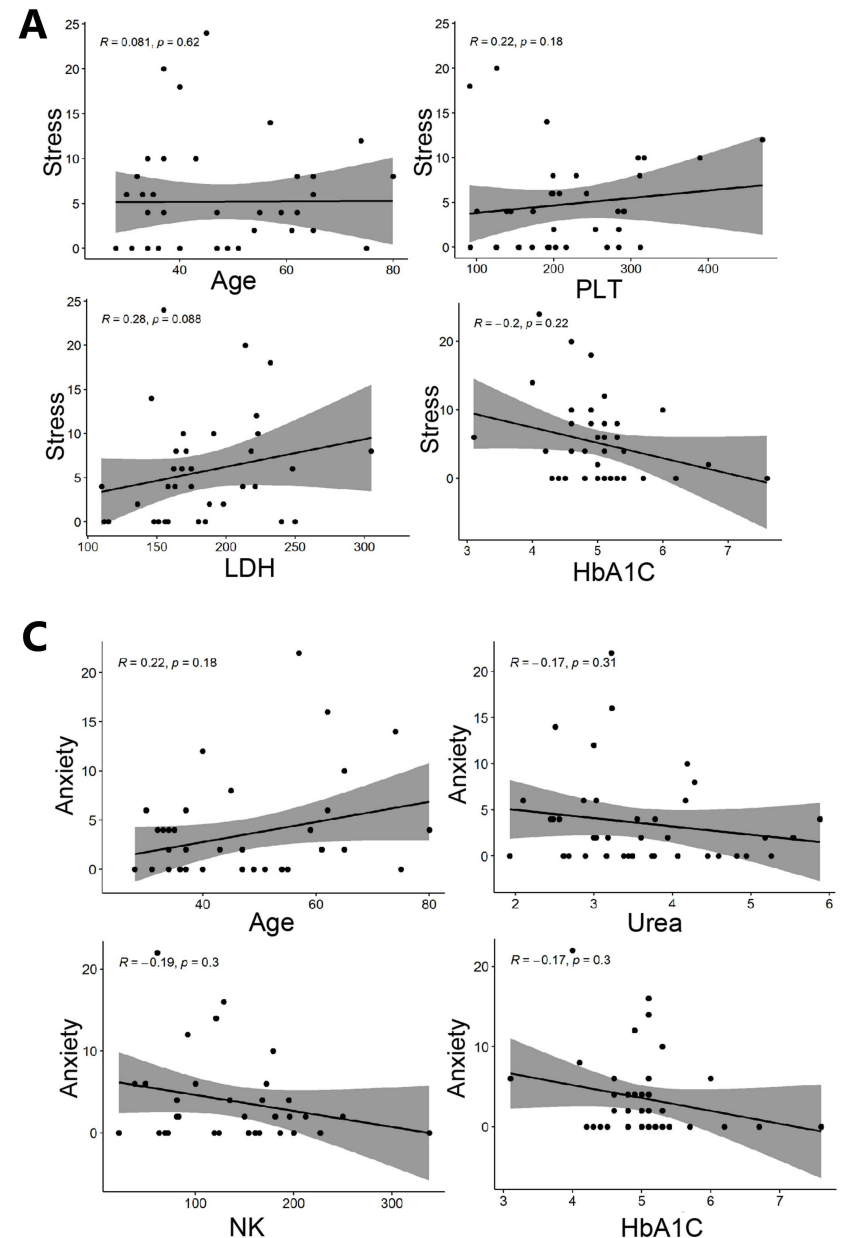
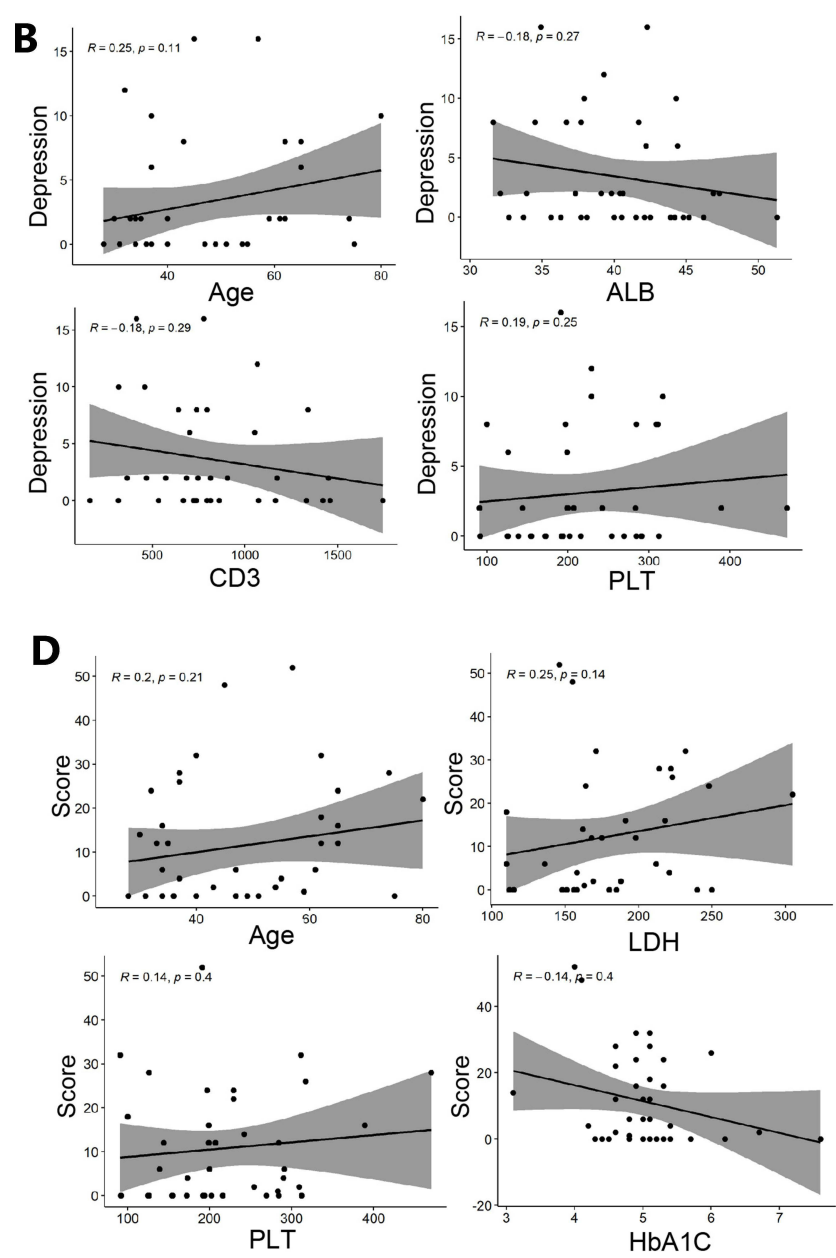

Figure 2 The scatter plot of Spearman correlation analysis of factors and psychological score (A) stress score and related factors; (B) depression score and related factors; (C) anxiety score and related factors; (D) total psychological score and related factors.

affecting the recovery of cardiac function were found, as shown in Table 3.

\section{Psychological Status of Patients with COVID-19 After 6-Month Discharge and Its Related Factors}

To estimate the psychological status after discharge, 40 patients completed the DASS- 21 scale 6 months after discharge. The cut-off scores of "mild", "moderate", "severe", and "extremely severe" depression, anxiety and stress were shown in Table S3. We found depression in 5 (12.5\%) participants, in which 2 $(5 \%)$ was at moderate level. Five $(12.5 \%)$ patients showed moderate or above anxiety, and $1(2.5 \%)$ of them showed extremely severe anxiety. Three (7.5\%) patients showed stress, and $2(5 \%)$ of them at moderate stage. However, only one patient with moderate depression and stress and mild anxiety was receiving antidepressant treatment.
Although no significant correlation factor was found, as shown in Table S4, some indicators were positively or negatively correlated with psychological scores, as shown in Figure 2. With the increase of age, depression, anxiety and total score have an increasing trend $(\mathrm{R}=0.25, \mathrm{P}=$ $0.113 ; \mathrm{R}=0.22, \mathrm{P}=0.182 ; \mathrm{R}=0.20, \mathrm{P}=0.210$, respectively). Compared with patients with antiviral therapy, patients without antiviral therapy had higher anxiety score ( 3 vs $0, \mathrm{P}=0.033$ ), as shown in Table S5.

\section{Discussion}

We found $11(22 \%)$ patients still presented symptoms after 6-month discharge. A previous study found $69 \%$ patients showed persisting symptoms 60 days after diagnosis of COVID-19 and $41 \%$ patients 100 days. ${ }^{14}$ The level of SARS-CoV-2 IgG was still elevated at the end of a 12week observation period. ${ }^{23}$ Three-month follow-up study found that SARS-CoV-2 IgG was still positive in $87.3 \%$ 
(48 of 55 patients) after discharge, while IgM was $12.7 \%$ (7 of 55 patients). ${ }^{13}$ In this study, the positivity of IgG was only $70 \%$ and $\operatorname{IgM}$ was $6 \%$, which was lower than the results in previous three-month follow-up study (IgG: $87.3 \%$, IgM: $12.7 \%){ }^{13}$ That indicated that the patient's symptoms continued to improve and the level of antibodies decreased over time.

Our study found that six months after discharge, the inflammatory index decreased and the immune function enhanced in patients with COVID-19. The values of CRP and SAA decreased significantly after discharge $(P<$ $0.001)$. The elevating cell counts of immunocytes, including CD3+ cells, B lymphocytes, NK cells, CD4+ T and CD8+ T lymphocytes, was observed 6 months after discharge in this study. In particular, the mean value of NK cell counts returned from below normal to normal (from 144.13 to 237.26 cells/ul, $P<0.001)$. Lymphocytopenia was present in $83.2 \%$ of patients after infection with SARS-CoV-2. ${ }^{24}$ In our previous study, we found the cell counts of these immunocytes were significantly decreased at admission. ${ }^{25}$ Acute SARS-CoV-2 infection impaired immune function of patients, nevertheless, the recovery of immune function played an important role in the rehabilitation of patients. ${ }^{26-28}$ In our study the elevating of NK cells was a good prognostic factor of pulmonary recovery.

The value of urea increased 6 months after discharge in our study, while creatinine did not show significant change. A previous 6-month follow-up study observed $479(35 \%)$ of 1378 patients with COVID-19 had decreased eGFR at follow-up. ${ }^{18}$ The percentage of HbAlc elevated 6 months after discharge. Interestingly, 4 patients showed normal $\mathrm{HbA1c}$ concentrations at discharge, but abnormal concentrations at 6-month follow-up, which was consistent with a previous 6-month follow-up study. ${ }^{18}$ Previous studies found diabetic individuals with uncontrolled glucose levels were more prone to develop the severe form of COVID-19. ${ }^{29,30}$ Although a in vivo study found SARSCoV-2 might not directly infect $\beta$ cells through ACE2 and TMPRSS $2,{ }^{31}$ new-onset diabetes and diabetic emergencies have been reported with COVID-19. ${ }^{32,33}$ We observed that the values of $\mathrm{CK}$ and $\mathrm{CK}-\mathrm{MB}$ elevated after discharge for 6 months, which might be ascribed to myocardial and skeletal muscle cell injury, such as myocarditis and myositis. ${ }^{34}$ With this in mind, the increase of these indicators needed the continuous attention of medical staff, though the values of $\mathrm{HbA1c}, \mathrm{CK}$ and CK-MB were still in the normal range after 6 months of discharge.
In this study, we found that the patients still had residual lung lesions 6 months after discharge, which was parallel to the previous study, which found that the patients had ground glass opacity and fibrosis 3 months after discharge. ${ }^{13,35}$ Previous study about severe acute respiratory syndrome (SARS) observed that at 6 months, 27.3\% patients showed reticulation and $72.7 \%$ showed GGO, while at 84 months, $72.7 \%$ patients showed reticulation and $18.2 \%$ showed GGO. ${ }^{36}$ In our study, comorbidities and age were risk factor for pulmonary recovery, while NK cell was a good prognostic factor. A previous 6-month follow-up study also found impaired pulmonary diffusion capacities were higher in patients with more severe illness. ${ }^{18}$ We found that compared with patients who did not receive antiviral treatment, treated patients had an increased proportion of lung consolidation disappearance and lower anxiety scores in psychological score 6 months after discharge. Nevertheless, previous clinical trials found antiviral drugs had little or no effect on hospitalized patients with COVID-19. ${ }^{37}$ No significant differences in viral clearance or clinical improvement were observed in COVID-19 patients treated with lopinavir/ritonavir. ${ }^{38}$ Oseltamivir has not been shown to have efficiency in COVID-19 treatment. $^{39}$ Darunavir/cobicistat did not increase the proportion of negative conversion vs standard of care alone. ${ }^{40}$ However, the long-term effect of antiviral drugs on patients with COVID-19 after discharge has not been reported. In the future, more studies are needed to explore the long-term effect of antiviral drugs on the prognosis of patients with COVID-19, so as to guide the correct clinical medication of patients.

A significant proportion of COVID-19 survivors had anxiety at discharge. ${ }^{41,42} \mathrm{~A}$ previous study indicated that $10 \%$ patients reported depression at one-month postdischarge. ${ }^{17}$ In this study, we found depression in 5 (12.5\%) participants, anxiety in $6(15 \%)$ patients, and stress in $3(7.5 \%)$ patients 6 months after discharge. With the increase of age, depression, anxiety and total score have an increasing trend, and compared with patients with antiviral therapy, patients without antiviral therapy had higher anxiety score. One previous study also found mental health problems were more prevalent among older adults, ${ }^{43}$ while several studies reported that younger individuals had a higher risk of anxiety. Thus, more studies are needed to clarify the influence of age in psychological status. $^{44,45}$ Antiviral therapy has also been found to decrease depression and anxiety in some chronic hepatitis C patients, ${ }^{46}$ while the mechanism was not clear. 
A previous study found that the methylprednisolone treatment might suppress anxiety through interactions between the locus coeruleus and hypothalamic-pituitary-adrenal axis in a rat model. ${ }^{47}$ Nevertheless, in this study, only 2 patients received methylprednisolone treatment, so we have not discussed its impact on the psychological state of patients. More studies are needed in the future to clarify the possible impact of methylprednisolone treatment on the psychological state of patients with COVID-19. Previous study reported that previous psychiatric illness history was a risk factor for anxiety. ${ }^{48}$ In this study, no patients had a history of psychiatric treatment, so its impact on severity of anxiety did not be discussed. A previous 6-month follow-up study found women had an OR 1.8 for anxiety or depression, ${ }^{18}$ while this sex difference was not observed in our study. Previous studies indicated that SARS survivors presented significant psychological distress at 1-month recovery and at 3-months after discharge, and still had elevated levels of psychological stress, after one-year outbreak. ${ }^{10,49-51}$ Therefore, the long-term psychological implications of SARS-CoV-2 infection should not be ignored.

SARS-CoV-2 infection could cause myocardial injury and diastolic dysfunction. ${ }^{52-54}$ In this study, echocardiography revealed abnormalities in $28(58 \%)$ patients in hospital, $24(52 \%)$ patients after 6-month discharge. Although no cardiac function was detected before SARSCoV-2 infection in this study, many previous studies have reported acute cardiac dysfunction after infection. ${ }^{52-54}$ Previous study has shown that patients with myocardial had a higher prevalence of hypertension, diabetes mellitus, and chronic kidney, but not prior myocardial infarction, prior stroke, history of atrial fibrillation and chronic obstructive pulmonary disease. ${ }^{53}$ Among the 32 patients without comorbidities in this study, $13(41 \%)$ patients showed abnormal echocardiography results, and 7 (22\%) patients showed left ventricular diastolic dysfunction. A previous study found the most common cardiac pathology within 24 hours of admission was right ventricular dilatation and dysfunction (observed in 39\% of patients), followed by left ventricular diastolic dysfunction (16\%). ${ }^{55}$ However, we found the most common pathology was left ventricular diastolic dysfunction (40\%) at discharge, which was $15 \% 6$ months after discharge. Previous study also reported cardiac involvement was observed in a proportion of recovering patients. ${ }^{56} \mathrm{~A}$ follow-up study indicated that the rate of diastolic dysfunction was observed in $60 \%$ patients on 60 days after diagnosis and $55 \%$ on 100 days. ${ }^{14}$ Therefore, follow-up studies were needed to explore the recovery of cardiac function in the process of rehabilitation.

This study had several limitations. We followed up patients with COVID-19 for 6 months and found that the immune function of the patients enhanced, but the impairments and impacts of SARS-CoV-2 infection on cardiopulmonary function, especially on psychology, continued to exist. However, the number of patients involved in our study was not large enough, and psychological impact was only assessed 6 months after discharge. We did not screen the comprehensive end organ, especially renal function, as well as lack of testing for neutralizing antibodies for SARS-CoV-2, the decline of which might be related to reinfection. ${ }^{18,57}$ Although we evaluated the risk factors of prognosis and found that age, comorbidities, NK cells, and antiviral therapy might be associated with the pulmonary recovery or psychological status, future studies covering larger sample size will be performed to further elucidate the validity.

\section{Conclusion}

In summary, we found that 6 months after discharge, inflammatory markers decreased and immune function enhanced in patients with COVID-19, while cardiopulmonary damage still lasted and psychological implications of SARS-CoV-2 infection should not be ignored. These results indicated that the sequelae of SARS-CoV-2 infection involves many aspects from body to mind, and lasts until 6 months after discharge. Therefore, longer and larger sample size follow-up studies are needed to understand the full spectrum of health consequences from SARS-CoV -2 infection.

\section{Data Sharing Statement}

The datasets used during this study are available from the corresponding author (Yilan Zeng) on reasonable request.

\section{Ethics Approval and Consent to Participate}

The study was conducted in accordance with the Declaration of Helsinki. This study has been approved by Ethics Committee of the Public Health Clinical Center of Chengdu (approval number: PJ-K2020-06-01) and registered at China Clinical Trial Registration Center (registration number: ChiCTR2000034563). Informed consent was obtained from each participation. 


\section{Acknowledgment}

Thanks to all people around the world who work so hard to fight against the COVID-19. Financial support. Shuiping Dai received grant support from the fellowship of China Postdoctoral Science Foundation (No. 2020M670054ZX), Weimin Li received support from the CAMS Innovation Fund for Medical Science (No: 2019TX310002), Yongzhao Zhou received support from the Sichuan Provincial Program for Diagnostic and Treatment of COVID-19 (No: 2020YFS0002), Yalun Li received support from the Sichuan Science and Technology Program (No. 2020YFS0572), Wenxin Luo received support from the major science and technology innovation project of Chengdu city (No. 2020-YF08 $-00080-G X)$.

\section{Author Contributions}

Shuiping Dai and Bennan Zhao: Writing - review and editing, Writing - original draft, Conceptualization, Project administration, Formal analysis, Methodology. Yongzhao Zhou: Project administration, Methodology, Writing - original draft. Yaling Liu: Data curation, Resources, Writing original draft. Lijuan Lan: Data curation, Formal analysis, Writing - original draft. Yalun Li and Wenxin Luo: Formal analysis, Methodology, Writing - review and editing. Yilan Zeng: Conceptualization, Supervision, Resources, Writing review and editing. Weimin Li: Conceptualization, Supervision, Resources, Funding acquisition, Methodology, Writing - review and editing. All authors made a significant contribution to the work reported, whether that is in the conception, study design, execution, acquisition of data, analysis and interpretation, or in all these areas; took part in drafting, revising or critically reviewing the article; gave final approval of the version to be published; have agreed on the journal to which the article has been submitted; and agree to be accountable for all aspects of the work

\section{Disclosure}

All authors report no conflicts of interest relevant to this article.

\section{References}

1. Zhu N, Zhang D, Wang W, et al. A novel coronavirus from patients with Pneumonia in China, 2019. N Engl J Med. 2020;382(8):727-733. doi:10.1056/NEJMoa2001017

2. Petersen E, Koopmans M, Go U, et al. Comparing SARS-CoV-2 with SARS-CoV and influenza pandemics. Lancet Infect Dis. 2020;20(9): e238-e244. doi:10.1016/S1473-3099(20)30484-9
3. Blanco-Melo D, Nilsson-Payant BE, Liu WC, et al. Imbalanced host response to SARS-CoV-2 drives development of COVID-19. Cell. 2020;181(5):1036-1045.e1039. doi:10.1016/j.cell.2020.04.026

4. Huang C, Wang Y, Li X, et al. Clinical features of patients infected with 2019 novel coronavirus in Wuhan, China. Lancet. 2020;395 (10223):497-506. doi:10.1016/S0140-6736(20)30183-5

5. Hu B, Guo H. Characteristics of SARS-CoV-2 and COVID-19. Nat Rev Microbiol. 2020;19:1-14.

6. Leung GM, Hedley AJ, Ho LM, et al. The epidemiology of severe acute respiratory syndrome in the 2003 Hong Kong epidemic: an analysis of all 1755 patients. Ann Intern Med. 2004;141(9):662-673. doi:10.7326/0003-4819-141-9-200411020-00006

7. Hui DS, Joynt GM, Wong KT, et al. Impact of severe acute respiratory syndrome (SARS) on pulmonary function, functional capacity and quality of life in a cohort of survivors. Thorax. 2005;60 (5):401-409. doi:10.1136/thx.2004.030205

8. Hui DS, Wong KT, Ko FW, et al. The 1-year impact of severe acute respiratory syndrome on pulmonary function, exercise capacity, and quality of life in a cohort of survivors. Chest. 2005;128 (4):2247-2261. doi:10.1378/chest.128.4.2247

9. Li SS, Cheng $\mathrm{CW}, \mathrm{Fu} \mathrm{CL}$, et al. Left ventricular performance in patients with severe acute respiratory syndrome: a 30-day echocardiographic follow-up study. Circulation. 2003;108(15):1798-1803. doi:10.1161/01.CIR.0000094737.21775.32

10. Wu KK, Chan SK, Ma TM. Posttraumatic stress, anxiety, and depression in survivors of severe acute respiratory syndrome (SARS). J Trauma Stress. 2005;18(1):39-42. doi:10.1002/jts.20004

11. Balachandar V, Mahalaxmi I, Subramaniam M, et al. Follow-up studies in COVID-19 recovered patients - is it mandatory? Sci Total Environ. 2020;729:139021. doi:10.1016/j.scitotenv.2020.139021

12. Xing Y, Mo P, Xiao Y, Zhao O, Zhang Y, Wang F. Post-discharge surveillance and positive virus detection in two medical staff recovered from coronavirus disease 2019 (COVID-19), China, January to February 2020. Euro Surveill. 2020;25(10). doi:10.2807/1560-7917. ES.2020.25.10.2000191

13. Liang L, Yang B, Jiang N, et al. Three-month follow-up study of survivors of coronavirus disease 2019 after discharge. J Korean Med Sci. 2020;35(47):e418. doi:10.3346/jkms.2020.35.e418

14. Sonnweber T, Sahanic S, Pizzini A, et al. Cardiopulmonary recovery after COVID-19 - an observational prospective multi-center trial. Eur Respir J. 2020;57:2003481.

15. An YW, Song S, Li WX, et al. Liver function recovery of COVID-19 patients after discharge, a follow-up study. Int J Med Sci. 2021;18 (1):176-186. doi:10.7150/ijms.50691

16. Qu G, Zhen Q, Wang W, et al. Health-related quality of life of COVID-19 patients after discharge: a multicenter follow-up study. J Clin Nurs. 2021;30(11-12):1742-1750.

17. Park HY, Jung J. Psychological consequences of survivors of COVID-19 Pneumonia 1 month after discharge. J Korean Med Sci. 2020;35(47):e409.

18. Huang C, Huang L, Wang Y, et al. 6-month consequences of COVID-19 in patients discharged from hospital: a cohort study. Lancet. 2021;397(10270):220-232. doi:10.1016/S0140-6736(20) 32656-8

19. Singhal T. A review of coronavirus disease-2019 (COVID-19). Indian J Pediatr. 2020;87(4):281-286. doi:10.1007/s12098-02003263-6

20. National Health Commission of the People's Republic of China. Diagnosis and Treatment Program of New Coronary Pneumonia. 7th ed. Beijing: Department of Medical Administration, Ministry of Health (MOH) of the PRC; 2020.

21. Antony MM, Bieling PJ, Cox BJ, Enns MW, Swinson RP. Psychometric properties of the 42-item and 21-item versions of the depression anxiety stress scales in clinical groups and a community sample. Psychol Assess. 1998;10(2):176-181. doi:10.1037/10403590.10.2.176 
22. Lu S, Hu S, Guan Y, et al. Measurement invariance of the depression anxiety stress scales-21 across gender in a sample of Chinese University students. Front Psychol. 2018;9:2064. doi:10.3389/ fpsyg.2018.02064

23. Li K, Huang B, Wu M, et al. Dynamic changes in anti-SARS-CoV-2 antibodies during SARS-CoV-2 infection and recovery from COVID-19. Nat Commun. 2020;11(1):6044. doi:10.1038/s41467020-19943-y

24. Guan WJ, Ni ZY, Hu Y, et al. Clinical characteristics of coronavirus disease 2019 in China. $N$ Engl J Med. 2020;382:1708-1720. doi:10.1056/NEJMoa2002032

25. Dai SP, Zhao X, Wu JH. Effects of comorbidities on the elderly patients with COVID-19: clinical characteristics of elderly patients infected with COVID-19 from Sichuan, China. J Nutr Health Aging. 2020;25:18-24.

26. Zhou R, To KK, Wong YC, et al. Acute SARS-CoV-2 infection impairs dendritic cell and $\mathrm{T}$ cell responses. Immunity. 2020;53 (4):864-877.e865. doi:10.1016/j.immuni.2020.07.026

27. Song JW, Zhang C, Fan X. Immunological and inflammatory profiles in mild and severe cases of COVID-19. Nat Commun. 2020;11 (1):3410

28. Yan L, Cai B, Li Y, et al. Dynamics of NK, CD8 and Tfh cell mediated the production of cytokines and antiviral antibodies in Chinese patients with moderate COVID-19. J Cell Mol Med. 2020;24:14270-14279.

29. Guo W, Li M, Dong Y, et al. Diabetes is a risk factor for the progression and prognosis of COVID-19. Diab/Metab Res Rev. 2020;36:e3319.

30. Jordan RE, Adab P, Cheng KK. Covid-19: risk factors for severe disease and death. BMJ. 2020;368:m1198. doi:10.1136/bmj.m1198

31. Coate KC, Cha J, Shrestha S, et al. SARS-CoV-2 cell entry factors ACE2 and TMPRSS2 are expressed in the microvasculature and ducts of human pancreas but are not enriched in $\beta$ cells. Cell Metab. 2020;32(6):10281040.e1024. doi:10.1016/j.cmet.2020.11.006

32. Hollstein T, Schulte DM, Schulz J, et al. Autoantibody-negative insulin-dependent diabetes mellitus after SARS-CoV-2 infection: a case report. Nat Metab. 2020;2(10):1021-1024.

33. Kim NY, Ha E. Acute hyperglycemic crises with coronavirus disease-19: case reports. Diab Metab J. 2020;44(2):349-353.

34. Kurapati R, Soos MP. StatPearls. Treasure Island (FL): StatPearls Publishing Copyright (C) 2021, StatPearls Publishing LLC.; 2021.

35. Zhao YM, Shang YM, Song WB, et al. Follow-up study of the pulmonary function and related physiological characteristics of COVID-19 survivors three months after recovery. EClinicalMedicine. 2020;25:100463. doi:10.1016/j.eclinm.2020.100463

36. Wu X, Dong D, Ma D. Thin-section computed tomography manifestations during convalescence and long-term follow-up of patients with Severe Acute Respiratory Syndrome (SARS). Med Sci Monit. 2016;22:2793-2799. doi:10.12659/MSM.896985

37. Pan H, Peto R, Henao-Restrepo AM, et al. Repurposed antiviral drugs for Covid-19 - interim WHO solidarity trial results. $N$ Engl $J$ Med. 2021;384(6):497-511.

38. Cao B, Wang Y, Wen D, et al. A trial of lopinavir-ritonavir in adults hospitalized with severe Covid-19. N Engl J Med. 2020;382 (19):1787-1799. doi:10.1056/NEJMoa2001282

39. Sanders JM, Monogue ML, Jodlowski TZ, Cutrell JB. Pharmacologic treatments for coronavirus disease 2019 (COVID-19): a review. JAMA. 2020;323(18):1824-1836.

40. Chen J, Xia L, Liu L, et al. Antiviral activity and safety of darunavir/ cobicistat for the treatment of COVID-19. Open Forum Infect Dis. 2020;7(7):ofaa241. doi:10.1093/ofid/ofaa241

41. Zhu S, Gao Q, Yang L, et al. Prevalence and risk factors of disability and anxiety in a retrospective cohort of 432 survivors of Coronavirus Disease-2019 (Covid-19) from China. PLoS One. 2020;15(12): e0243883. doi:10.1371/journal.pone.0243883
42. Cai X, Hu X, Ekumi IO, et al. Psychological distress and its correlates among COVID-19 survivors during early convalescence across age groups. Am J Geriatr Psychiatry. 2020;28(10):1030-1039. doi:10.1016/j.jagp.2020.07.003

43. Chew NWS, Lee GKH, Tan BYQ, et al. A multinational, multicentre study on the psychological outcomes and associated physical symptoms amongst healthcare workers during COVID-19 outbreak. Brain Behav Immun. 2020;88:559-565. doi:10.1016/j.bbi.2020.04.049

44. Huang Y, Zhao N. Generalized anxiety disorder, depressive symptoms and sleep quality during COVID-19 outbreak in China: a web-based cross-sectional survey. Psychiatry Res. 2020;288:112954. doi:10.1016/j.psychres.2020.112954

45. Wang Y, Di Y. Study on the public psychological states and its related factors during the outbreak of coronavirus disease 2019 (COVID-19) in some regions of China. Psychol, Health Med. 2021;26(1):13-22.

46. Durcan E, Hatemi I, Sonsuz A, Canbakan B, Ozdemir S, Tuncer M. The effect of direct antiviral treatment on the depression, anxiety, fatigue and quality-of-life in chronic hepatitis $\mathrm{C}$ patients. Eur J Gastroenterol Hepatol. 2020;32(2):246-250. doi:10.1097/ MEG.0000000000001501

47. Tanriverdi AM, Aydin B, Bebitoglu BT, Cabadak H, Goren MZ. The behavioral and neurochemical effects of methylprednisolone or metyrapone in a post-traumatic stress disorder rat model. North Clin Istanbul. 2019;6(4):327-333.

48. Özdin S, Bayrak Özdin Ş. Levels and predictors of anxiety, depression and health anxiety during COVID-19 pandemic in Turkish society: the importance of gender. Int J Soc Psychiatry. 2020;66 (5):504-511. doi:10.1177/0020764020927051

49. Lee AM, Wong JG, McAlonan GM, et al. Stress and psychological distress among SARS survivors 1 year after the outbreak. Can $J \quad$ Psychiatry. 2007;52(4):233-240. doi:10.1177/07067437 0705200405

50. Cheng SK, Wong CW, Tsang J, Wong KC. Psychological distress and negative appraisals in survivors of severe acute respiratory syndrome (SARS). Psychol Med. 2004;34(7):1187-1195. doi:10.1017/ S0033291704002272

51. Kwek SK, Chew WM, Ong KC, et al. Quality of life and psychological status in survivors of severe acute respiratory syndrome at 3 months postdischarge. J Psychosom Res. 2006;60(5):513-519. doi:10.1016/j.jpsychores.2005.08.020

52. Qian SZ, Hong WD, Lingjie M, Chenfeng L, Zhendong F, Pan JY. Clinical characteristics and outcomes of severe and critical patients with 2019 novel coronavirus disease (COVID-19) in Wenzhou: a Retrospective Study. Front Med. 2020;7:552002. doi:10.3389/ fmed.2020.552002

53. Giustino G, Croft LB, Stefanini GG, et al. Characterization of myocardial injury in patients with COVID-19. J Am Coll Cardiol. 2020;76(18):2043-2055. doi:10.1016/j.jacc.2020.08.069

54. Balaban Kocas B, Cetinkal G, Ser OS, et al. The relation between left ventricular global longitudinal strain and troponin levels in patients hospitalized with COVID-19 pneumonia. Int J Cardiovasc Imaging. 2020;37:1-9.

55. Szekely Y, Lichter Y, Taieb P, et al. Spectrum of cardiac manifestations in COVID-19: a Systematic Echocardiographic Study. Circulation. 2020;142(4):342-353. doi:10.1161/CIRCULATION AHA.120.047971

56. Huang L, Zhao P, Tang D, et al. Cardiac involvement in patients recovered from COVID-2019 identified using magnetic resonance imaging. JACC Cardiovasc Imaging. 2020;13(11):2330-2339. doi:10.1016/j.jcmg.2020.05.004

57. Wajnberg A, Amanat F. Robust neutralizing antibodies to SARS-CoV-2 infection persist for months. Science. 2020;370 (6521):1227-1230. 


\section{Publish your work in this journal}

The International Journal of General Medicine is an international, peer-reviewed open-access journal that focuses on general and internal medicine, pathogenesis, epidemiology, diagnosis, monitoring and treatment protocols. The journal is characterized by the rapid reporting of reviews, original research and clinical studie across all disease areas. The manuscript management system is completely online and includes a very quick and fair peer-review system, which is all easy to use. Visit http://www.dovepress.com/ testimonials.php to read real quotes from published authors.

Submit your manuscript here: https://www.dovepress.com/international-journal-of-general-medicine-journal 